\title{
Research on steel template design for supporting system of large cantilever special-shaped concrete structure
}

\author{
Yan Zhou, Jian Cao* ${ }^{*}$ Linna $\mathrm{Hu}$ and Cheng $\mathrm{Hu}$ \\ School of Civil Engineering and Architecture, Nanchang Institute of Technology, Nanchang, Jiangxi, 330099, China
}

\begin{abstract}
This paper analyzes the difficulties of steel template design. BIM information model was used for reference to set up a three-dimensional model. Structure safety, forming quality and construction convenience were fully considered. This paper takes Nanchang Chaoyang bridge as an example for the work of template design in each stage. By exploring the selection criteria of shaped structural steel template and establishing the corresponding calculation and design method, the implementation quality of the bridge tower was ensured. Research formed a template design system of template calculation and design. This system provided reference for other similar types of special structure template design.
\end{abstract}

\section{Introduction}

Because of full space tension, beauty, and metre of special-shaped structure, it is widely used in the engineering field and has accumulated a lot of engineering practice experience ${ }^{[1]}$. However, as the large cantilever special-shaped concrete structure has strong uncertainty in the aspects of lofting, steel bar positioning, template making, template assembling and casting deformation control, there are also great challenges in the construction. The reasonable design and excellent production of steel template of supporting system of large cantilever special-shaped concrete structure are the foundations of the bridge tower construction ${ }^{[2]}$. In order to guarantee the quality of the bridge tower, it is necessary to research the selection standard of special-shaped structure steel template and establish corresponding calculation and design method ${ }^{[3]}$.

This paper takes Nanchang Chaoyang bridge as an example and uses BIM information model in existing engineering practice for reference. Three-dimensional model is also used to carry out the template design of each section. The research forms a template design system that integrates template calculation and design. This study also provides reference for other similar special-shaped structure template design.

\subsection{Project summary}

Chaoyang bridge (Figure 1) is located between Nanchang bridge and Shengmi bridge (about $2.5 \mathrm{~km}$ away from Nanchang bridge and $3.5 \mathrm{~km}$ away from Shengmi bridge), connecting Chaoyang new area of Nanchang and Red-state district.

The mainspan of Chaoyang bridge adopt a single cable plane low pylon with six towers and seven openings cable-stayed bridge scheme. Main girder adopts steel concrete composite beam. The bridge across Ganjiang river and the total length is about 1560 meters and is divided into two parts: the main bridge and the approach bridge. Pylon model of Chaoyang bridge is deformation of the calligraphy word "Unity", which softens the word line of classic small seal "Unity" and the whole model's curve is soft vivid fluent. The design of hollow mix light and space together well, which gives expression to the idea of "Great Harmony" that encompassing universal phenomena and taking in diversity. However, special-shaped structure also raise the construction difficulty. The construction technology and quality control becomes the key issue in the construction of pylon and pier.

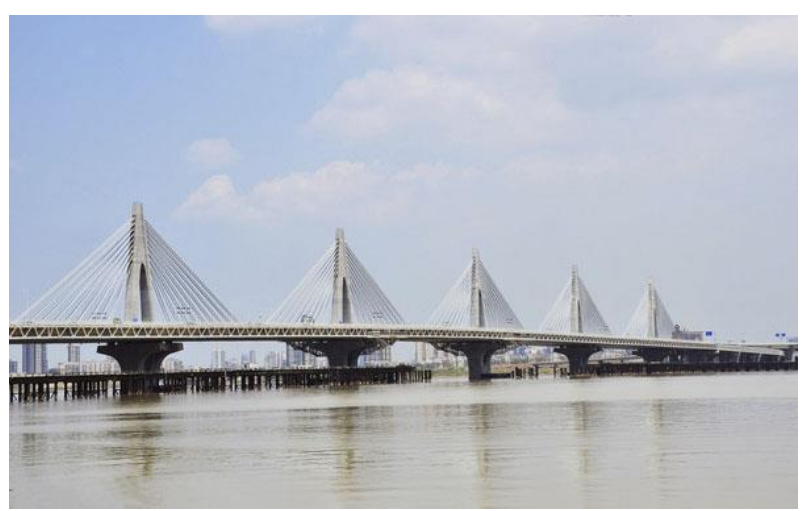

Figure 1. Nanchang Chaoyang Bridge.

\section{Case Analysis}

\section{I. Difficulties of steel template design}

For the supporting system of special-shaped concrete structure, the difficulties of template design is mainly 
caused by the highly warping of the bridge tower space, Difficulties mainly reflect in the aspect of expression of template surface, design adjustment and optimization and template component design.

\subsubsection{Difficulties in expression of template surface.}

Taking Nanchang Chaoyang bridge as an example (Figure 2), the design configuration of its lower pylon and upper pylon is relatively complicated.

The hollow of lower pylon Longitudinal bridge is

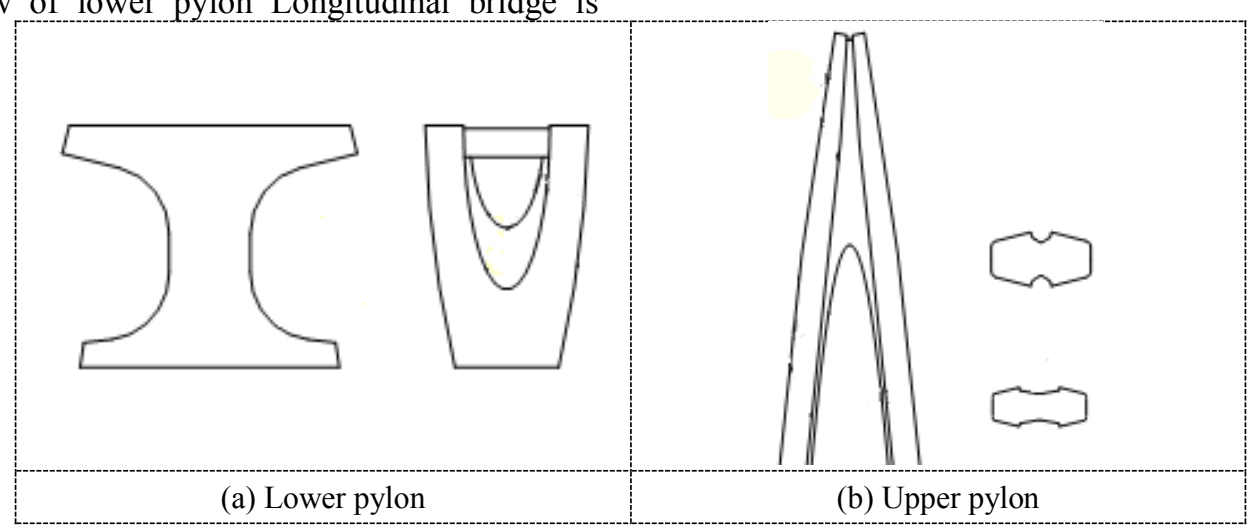

Figure 2. Spatial surface analysis of Nanchang Chaoyang bridge pylon.

\subsubsection{Difficulties in design adjustment and optimization.}

In the template design, segmentation adjustments is necessary, and it is advisable to optimize the setting of the template. If the segmentation position is obtained by calculation each time, it will take huge workload. Prone to errors or omissions, which will affect the template making and installation and even cause rework, bringing great economic losses.

\subsubsection{Difficulties in template components design.}

The longitudinal and longitudinal steel bands stiffening I rib, connecting hole plate and back plate stiffening etc. on the template are all extended outward based on curved surface shape. Under the complex expression of the template surface, it is difficulty to carry out the accurate design of the template components and affect the assembly accuracy of each component.

\subsection{Methods of design and calculation}

Due to the remarkable characteristics of the template space, the method of traditional calculation and analysis may hard to satisfy the complex calculation requirements, so unique calculation ideas and methods must be established.

\subsubsection{Simulation mode.} local components, it is necessary to distinguish their stress modes and simplify them by different methods. For the lower pylon with the most complex space
According to the simulation of the template system and composed of arc lines and straight lines, while from the transverse bridge view the both sides of curve is divided by oval line and arc line. The outer line of upper pylon is an arc line, while the inner line is joint by two arc lines. The inner of points limb is configurated by parabola. It can be seen from the profile that the above line shape is just the totality control linearity, while several changeable arc lines chamfering exist on the section. It is difficult to express each side of the upper and lower pylons by simple formula. configuration, three-dimensional modeling method should be adopted. Different elements should be adopted to simulate the steel plate, back beam and tie bar. The steel plate adopts space shell element, the back beam adopts space beam element, and the tie bar adopts bar element.

\subsubsection{Loading mode.}

In the analysis and calculation, the types of value and criteria of the evaluation that template system bore during the whole construction should be entirely considered. Combination of unfavorable load on the load range during construction period should be taken into consideration, followed are the complexity of calculation and checking calculation of main stress condition. According to the analysis, load mainly concerns structure gravity, flow plasticity pressure, temporary effect of machine on the template and so on.

\subsubsection{Selection of calculation software.}

Calculation software should have strong space force analysis ability, which covers various types of unit simulated by various units. Because orthotropic deck has more components, analysis software should have strong previous processing functions. Based on the requirements of template space calculation, lower pylon calculation chooses SolidWorks 2011. SolidWorks have powerful functions and various components. SolidWorks has three characteristics, which are powerful functions, easily learned and applying and technology innovation. SolidWorks is a convenient software which integrates calculation, design and plot into itself. 


\subsubsection{Check calculation content.}

According to the basic principles of the template design, structure safety and concrete pouring quality must be guaranteed, which means template should satisfy the requirements that stress safely and deformation meets concrete structure deviation. Therefore, subject check calculation content are component stress and deformation [4].

\section{Calculation and design of lower pylon steel template}

\subsection{Calculation of lower steel template}

Taking lower pylon's first casting template model as an example. Three dimensional model was built by SolidWorks, and then details of the structure was simplified. Remove some features and parts that have little influence on stress check calculation, and take the symmetry of the simplified model into consideration. 1/4 model was used for analysis in actual operation to reduce calculation amount and improve the calculation accuracy. Diagrammatic figure as Figure 3.

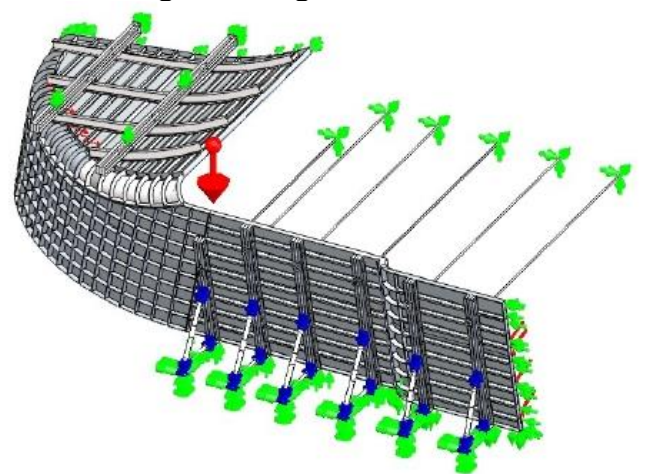

Figure 3. First casting template model and constraint of lower pylon.

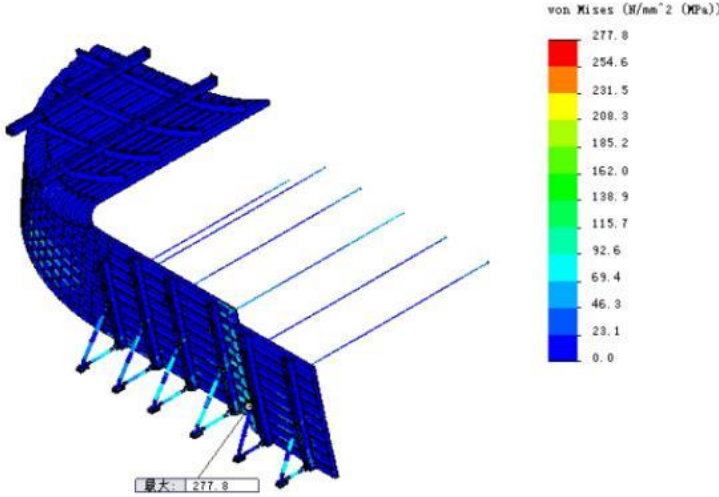

Figure 4. Stress analysis of first casting template.

The maximum stress of first casting section is $277.8 \mathrm{MPa}$, which occurs at intersection of transverse stiffening plate and vertical stiffening plate in the cross the bridge plate. According to elastic-plasticity theory, this stress is local stress, most of which under $140 \mathrm{MPa}$.

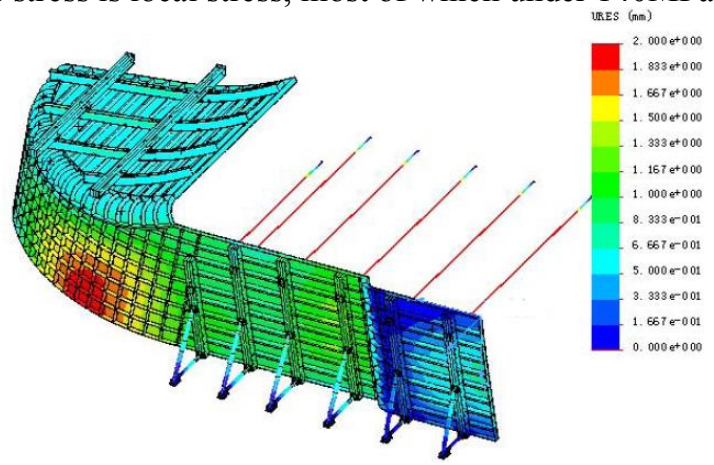

Figure 5. Deformation of first casting template.

\subsection{Lower pylon steel template design}

The height of a single template shall not be greater than $3 \mathrm{~m}$. Reinforcing ribs arrange transversely while back beams were arranged vertically. Back beams that follow the bridge designed as truss form. For the convenience of template installation, spiral supporting poles were set on the cushion cap under the transverse and longitudinal direction of bridge template.
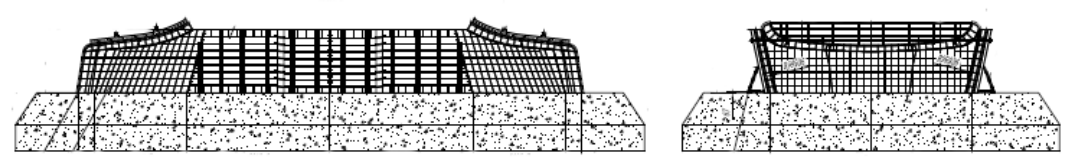

Figure 6. Template design of first casting template.

In both transverse and longitudinal direction of bridge, pull rods are arranged evenly with spacing between $1 \mathrm{~m}$ and $1.2 \mathrm{~m}$. If the pull rods interfere with rebars or stiff skeletons, withdraw the position of rebars or stiff skeletons suitably.

Because lower pylon is big in the bottom while small in the top. There is a work window near the bottom of longitudinal direction of first casting. In addition, in order to prevent templates which are longitudinal direction of the bridge floating up, the tie rods need to be installed after the sleeves were embedded on the top of the pile caps in advance.
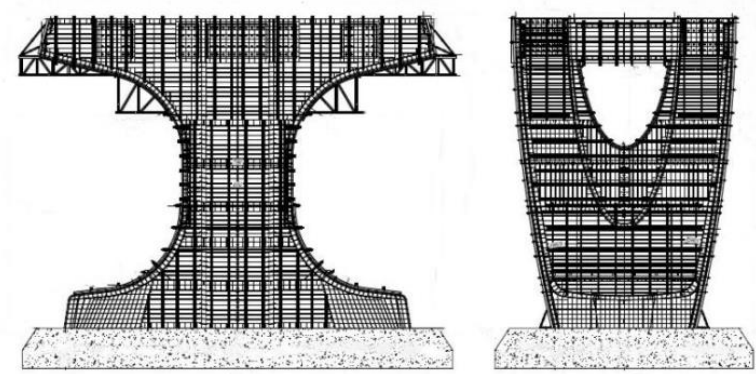

Figure 7. Template structure of lower pylon. 


\subsection{Total design of steel template}

The lower pylon cast in four times, the calculation and design of first casting template are given above. The calculation and design of last three casting templates could obtained by the same method. The overall blue print of final template as Figure 7.

\section{Conclusion and discussion}

Method of BIM information model in existing engineering practice, which can not only be applied to template design, but also it can control processing manufacture、 lofting of template.

Three advantages of adopting three-d model as follow. Firstly, it could guarantee the accuracy of templates. For special-shaped component, model information is the basic of all information, the veracity and accuracy of which must be guaranteed. Powerful model software is adopted in this process to solve the modeling, plot and calculation of complex special-shaped structure. The process of model making and revise must be strictly controlled to guarantee the accuracy of template. Secondly, it could realize the functions of segmentation, map and so on. Adopting three-d model could realize the functions of segmentation and map, which is conducive to the transformation of information models into several key acceptable two-dimensonal information models. Thirdly, it could embed into installation process. During the establishment of model, the template installation process is taken into consideration and the visualization of construction is realized.

However, in the application of BIM technology in the project, the following deficiencies remain. Firstly, design method of existing BIM exists some deficiencies: single modeling method, lack of intelligence and lack of professionalism. Secondly, special-shaped structures in existing project often occur in terms of bridges. Existing BIM has difficulties in modeling.

Here proposes items that need deeper research in BIM technology in next stage. Firstly, gradually deepening the bridge information model modeling theory. Secondly, the improvement of steel structure checking method based on design, manufacture and operation process. Parametric reinforcement design method based on concrete structure surface and mass need to be improved as well. Thirdly, on account of requirement, establishing an operation stage that faces bridge major professionals, which could optimize the professional drawing function and realize software friendly upgrade.

\section{Acknowledgements}

The authors would like to acknowledge the financial support of the Science and Technology Project Founded by the Education Department of Jiangxi Province with Grant No. GJJ170985 and the Natural Science Foundation of Jiangxi Province of China with Grant No. 20161BAB206120.

\section{References}

1. Ou, Y.S. (2017) Optimal Design and Quality Control of Bridgepier Steel Formwork. Mechanical Engineer. J, 2: 126-128.

2. Huang, T., Lu, W.L., Gou, T.T., Kan, L.C. (2016) Experiment Study on Formwork Design of Precast Segmental Bridge Pier. Construction Technology. J, 45(11): 1-3.

3. Yuan, J.X. (2012) The study on the Application of the Construction Technique for Bridge Formwork. ShanXi Science \& Technology of Communications. J, 4: 27-29.

4. Editiorial Department. (2016) BIM Application of Chaoyang Bridge in Nanchang. Construction Quality. J, 34: 88-92. 